\title{
Capillary-valve-based platform towards cell-on-chip mechanotransduction assays
}

\author{
Tanja C. Hausherr ${ }^{\mathrm{a}, \mathrm{b}, *}$, Hicham Majd ${ }^{\mathrm{b}}$, Damien Joss ${ }^{\mathrm{a}, \mathrm{b}}$, Arnaud Müller $^{\mathrm{a}}$, \\ Dominique P. Pioletti ${ }^{b}$, Martin A.M. Gijs ${ }^{a}$, Christophe Yamahata ${ }^{a}$ \\ a Laboratory of Microsystems, Institute of Microengineering, École Polytechnique Fédérale de Lausanne (EPFL), Lausanne, Switzerland
b Laboratory of Biomechanical Orthopedics, Interinstitutional Center of Translational Biomechanics, École Polytechnique Fédérale de Lausanne (EPFL),
Lausanne, Switzerland
}

\section{A R T I C L E I N F O}

\section{Article history:}

Received 19 March 2013

Received in revised form 13 June 2013

Accepted 15 July 2013

Available online $\mathrm{xxx}$

\section{Keywords:}

Capillary valve

Bio-microelectromechanical system

(bio-MEMS)

Silicon microchip

Mechanotransduction

Cell assay

\begin{abstract}
A B S T R A C T
Reliable in vitro models are required to understand the ability of cells to respond and adapt to mechanical stimuli. To mimic and interface with the microenvironment, lab-on-a-chip devices and microelectromechanical systems (MEMS) provide excellent options. However, little effort has been done in combining them. To address this shortcoming, we have developed a versatile microengineered platform which consists of two parts: an electrostatically actuated MEMS device used for mechanobiology assays, and a fluidic system for cell culture. A capillary valve allows inserting a silicon chip horizontally in the culture medium without leakage and without wetting of the electrostatic microactuators. The platform is designed for mechanotransduction assay on cells and aims specifically human mesenchymal stem cells. The proof of principle of the platform was performed by stable and long-term cultures of rat fibroblasts. We could also study the effect of periodic stress at various excitation frequencies.
\end{abstract}

(c) 2013 Elsevier B.V. All rights reserved.

\section{Introduction}

Cells have the capacity to adapt to dynamic changes in their natural microenvironment. In particular, cells can sense and convert mechanical forces into chemical signals via mechanotransduction [1]. The study of those mechanical stimuli and responses, defined as mechanobiology, has led to numerous in vitro studies controlling the micromechanical environment [2,3]. Multipotent mesenchymal stromal cells (MSC) are the most studied stem cells due to their role in the formation and maintenance of load-bearing tissues in the musculoskeletal system. Thus, they are very attractive for tissue engineering and cell therapy [4]. Even though it is well known that the environment influences the fate of stem cells, still very little is known about the relative importance of these environmental factors. Different tools and strategies have been shown to induce MSC differentiation by mechanical means, such as inplane substrate distension [5], lineage differentiation upon stretch

\footnotetext{
* Corresponding author at: Laboratory of Biomechanical Orthopedics, Interinstitutional Center of Translational Biomechanics, École Polytechnique Fédérale de Lausanne, CH-1015 Lausanne, Switzerland. Tel.: +41 2169383 32; fax: +41216938660.

E-mail addresses: tanja.hausherr@epfl.ch (T.C. Hausherr), christophe.yamahata@a3.epfl.ch (C. Yamahata).
}

[6], and early lineage differentiation of cells according to the stiffness of their natural environment [7]. While it is clearly accepted that the conjugation of all these factors influences the differentiation of MSC, the importance of each isolated factor remains to be elucidated.

In recent years, microtechnologies have enabled novel experimental capabilities for (i) the measurement of mechanical properties of cells, nuclei, cell membranes and cytoskeleton, and (ii) the study of the reaction of cells to specific mechanical stresses, such as micropipette aspiration, extracellular matrix microcontact printing or micromechanical cell stretching $[1,8,9]$. Thanks to biological microelectromechanical systems (MEMS/bio-MEMS) tools, more accurate manipulation on cells, as well as quantitative measurements of cellular responses with high spatial and temporal resolution are possible.

For single cell mechanobiology studies, the combination of fluidics with a microactuator is thought to be a promising alternative to tedious instrumentation currently used by biophysicists (e.g., optical tweezers, micropipetting, AFM indentation). However, such an approach implies jointly developing the fluidic system and the MEMS device, in addition to working on the interface between immersed and actuated parts. In that matter, one tool that caught our attention is that of Yang and Saif who have used an AFM-like silicon probe for indentation assays on living cells [10]. In the proposed setup, the sensor plane is inclined by few degrees to prevent 
contact with the bottom of the culture dish. Thus, it cannot be used to adhere cells directly to the silicon substrate: to ensure homogeneous cell sedimentation, the substrate must remain horizontal. The latter issue was addressed in a device presented by Serrel et al. for uniaxial tensile assays on cells [11]. However, while a silicon substrate was used for these experiments, an additional external probe was also used to actuate the device. Taking full advantage of microfabrication technologies, the afore mentioned issues could be addressed by researchers at IMEC, Belgium. They have developed an encapsulation technique that enables MEMS devices to be operated in aqueous environment, as they could demonstrate with an electrostatically actuated inchworm [12]. The basic idea behind this technique consists in making the surfaces hydrophobic at the clearance, where the actuator shuttle extends off the device [13].

Rather than rendering the silicon chip microfabrication more complex, capillary action can also be used judiciously to interface a fluidic system with a silicon device [14,15]. It is on this basis that we have developed our platform dedicated to the study of the influence of mechanical stress (e.g., stretching and compression) on cell differentiation. As cell-on-chip mechanical assays must accommodate the stringent requirements for cell culture, we have opted for the design of a microengineered platform consisting of an electrostatically actuated silicon chip and an open fluidic system. A passive capillary valve was chosen to allow for a partial horizontal immersion of our MEMS device in the culture medium. We draw the reader's attention to the fact that the biological experiments discussed in this paper form a pre-study for the validation of our device and were performed on rat fibroblasts (RFB). Those cells are known to differentiate into myofibroblasts under mechanical stress [16].

\section{Materials and methods}

\subsection{Fabrication}

The chips were microfabricated using silicon-on-insulator technology, following a three-mask process similar to that presented in ref. [17]. Micromachining was performed on a (100)-oriented SOI wafer having a resistivity in the range of $1-10 \Omega \mathrm{cm}$, a $50 \mu \mathrm{m}$ thick device layer, a $2 \mu \mathrm{m}$ thick buried oxide layer, and a $380-\mu \mathrm{m}$ thick handling substrate. First, a $100-n m$ thick aluminium film was evaporated on the device layer. After a first frontside photolithography (mask No. 1 defining the electrical pads for wire bonding), aluminium was patterned using an inductively coupled plasma system. Subsequently, a second photolithography (mask No. 2) was performed on the frontside and the silicon device layer was etched by deep reactive ion etching (DRIE) using the Bosch process. After resist removal, the wafer backside was patterned with a thick photoresist (mask No. 3). To protect the device layer and strengthen the wafer, a $2 \mu \mathrm{m}$ thick parylene conformal coating was deposited on the frontside. Then, etching of the handling substrate was performed by DRIE (with high etch rate compared with frontside etching) using the Bosch process. Finally, after resist and parylene removal by plasma etching, HF vapor was used to remove the buried oxide beneath the moving structures. Chip-on-board packaging on printed circuit boards (PCB) was achieved by aluminium wedge-bonding and glob-top encapsulation in an epoxy resin.

Regarding the various elements composing the fluidic platform, all the materials were carefully chosen to ensure compatibility with conventional sterilization techniques (namely, autoclaving, $70 \%$ ethanol disinfection or UV germicidal irradiation). The culture chamber and its protection lid were machined in polycarbonate (PC). For firm assembly of the silicon chips to the setups and their connection to electronic instruments, the PCB were screwed by means of PC parts containing all the electrical plugs. To facilitate centering and insertion of the MEMS device into the fluid compartment, a holder and a sliding mechanism were designed in PC and in teflon. Partial immersion of the silicon chip in liquid could be controlled with the naked eye thanks to a blue-coloured valve moulded with a mixture of polydimethylsiloxane (PDMS, Sylgard 184 silicon elastomer, ratio 1:10, Dow Corning, USA) and commercially available ink. Also, to perform several cell assays simultaneously, three identical platforms were fabricated on which the disposable PDMS valves were systematically replaced after each experiment.

\subsection{Sterilization and functionalization}

The packaged chips were autoclaved at $100^{\circ} \mathrm{C}$ for $10 \mathrm{~min}$. The platforms and PDMS valves were sterilized in $70 \%$ ethanol for $2 \mathrm{~h}$ and dried $1 \mathrm{~h}$ under laminar flow. For surface functionalization, $100 \mu \mathrm{L} / \mathrm{mL}$ fibronectin (Invitrogen, USA) in phosphate buffered saline (PBS, pH7.4, Invitrogen) were incubated on the silicon devices for $1 \mathrm{~h}$ at room temperature (RT). The chips were consecutively washed with PBS.

\subsection{Cell culture}

RFB were obtained by tissue extraction [18] and were kept in filtered Dulbecco's modified Eagle medium (DMEM, Invitrogen) supplemented with $10 \%$ fetal calf serum (FCS, Thermo Scientific, USA), 1\% L-glutamine, 1\% penicillin-streptomycin (Invitrogen) at $37^{\circ} \mathrm{C}$ and $5 \% \mathrm{CO}_{2}$. Experiments were performed between passages 3 and 4 with a seeding density of 10,000 cells $/ \mathrm{cm}^{2}$, in both the platforms and the polystyrene Petri dishes used as controls.

\subsection{Immunohistochemistry}

RFB were fixed with 4\% filtered paraformaldehyde (PFA, Sigma-Aldrich, Switzerland) for $10 \mathrm{~min}$, then permeabilised in $0.2 \%$ Triton X-100 (Applichem, Switzerland) in PBS for 10 min at RT. Cells were subsequently washed extensively with PBS. For fibronectin detection, cells were stained with the primary antibody F3648 antifibronectin (Sigma-Aldrich) for $1 \mathrm{~h}$. The secondary antibody Alexa 647 (Invitrogen) was simultaneously incubated for $1 \mathrm{~h}$ in the dark with the cell nuclei and F-actin stains, DAPI (Invitrogen) and phalloidin Alexa 488 (Invitrogen), respectively. After PBS washing, RFB were analysed by confocal microscopy (LSM 700, Zeiss, Germany).

\subsection{Statistical analysis}

Data were analysed using the Tukey's test for which a $P$ value $<0.05$ was accepted as statistically significant. Each time an experiment was launched, three microchips were simultaneously analysed (one per platform), of which one served as control and was used without coating nor actuation. Each experiment was repeated three times to ensure consistency of the results. Each data point in Fig. 5 represents the mean value plus or minus one standard deviation calculated from these measurements.

\section{Results and discussion}

\subsection{Open fluidic platform}

The working principle of our open fluidic platform is depicted in Fig. 1A. Thanks to the principle of the passive capillary valve, the silicon microchip is partially immersed in the culture medium while its comb-drives are kept dry. The immersed part of the chip consists of fixed and mobile plates. The mobile plates are electrostatically actuated and move back and forth between the immobile plates. After cell seeding, uniform deposition of cells occurs. In this research, we 
A

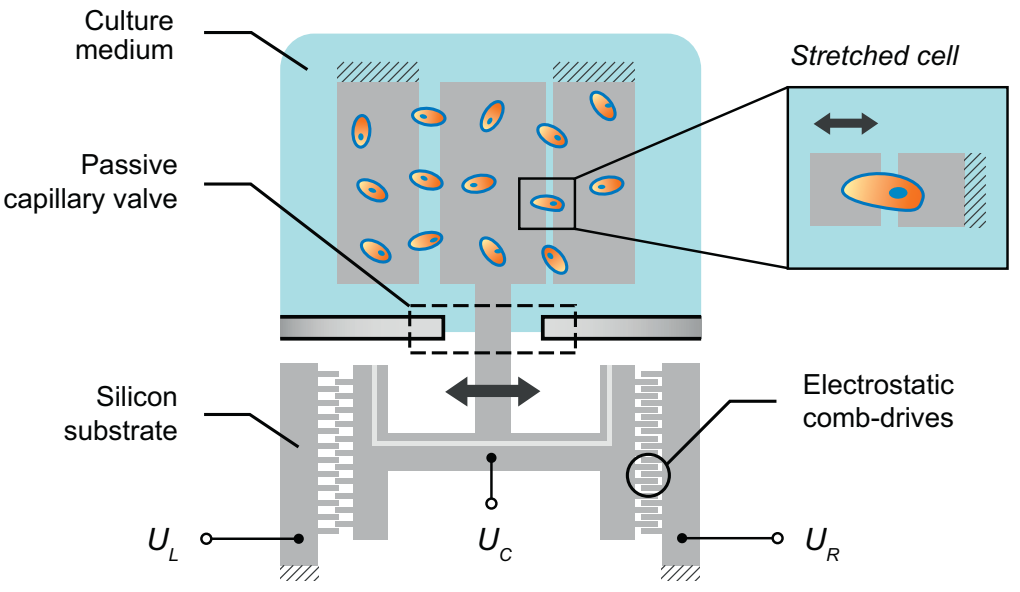

B

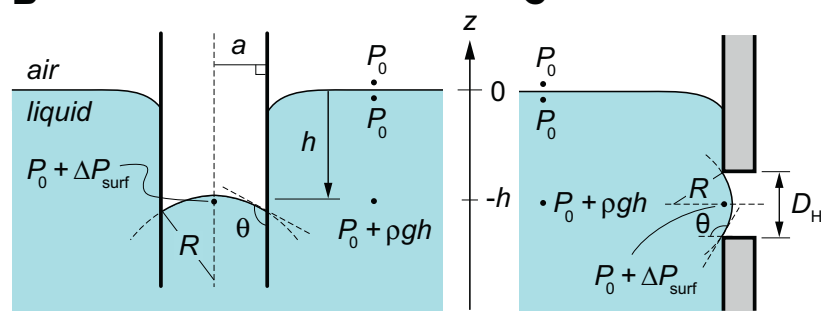

D

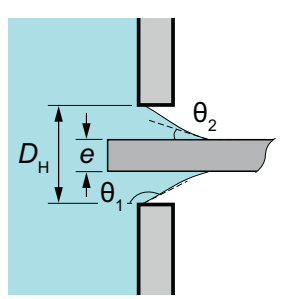

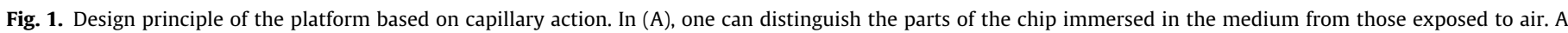

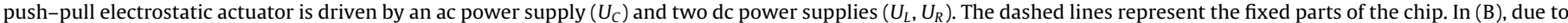

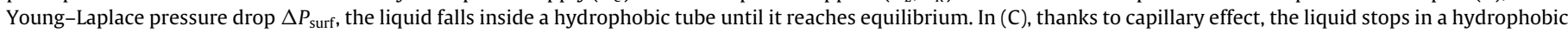
side aperture. In (D), we show equilibrium state after a thin plate has been introduced in the capillary valve.

are interested in the impact of stretching and compression of cells bridging between the immobile and mobile plates.

Leak-free insertion of the MEMS device into the side opening of the fluidic system is based on capillary action, as illustrated in Fig. 1B-D. Capillary effects are significant at length scales smaller than the capillary length, $\lambda_{c}$, defined as [19]

$\lambda_{c}=\sqrt{\frac{\gamma}{\rho g}}$,

where $\gamma$ is the surface tension of the liquid/air interface $\left(\gamma=0.07 \mathrm{~J} \mathrm{~m}^{-2}\right.$ for a water/air interface at $\left.37^{\circ} \mathrm{C}\right), \rho$ is the liquid density and $g$ is Earth's gravity. For water, the capillary length is $\lambda_{c} \approx 2.7 \mathrm{~mm}$. Hence, for a thin and wide rectangular aperture of height $d=1 \mathrm{~mm}$ (width $w \gg d$ ), the hydraulic diameter is $D_{\mathrm{H}}=$ $2 \frac{w d}{w+d} \approx 2 d=2 \mathrm{~mm}$, and gravity does not significantly influence the shape of the free water/air interface [19].

Let us consider capillary action that happens in a narrow, vertically standing microtube with a circular cross-section of diameter $2 a<\lambda_{c}$, as depicted in Fig. 1B. The liquid/air interface is curved, which causes a Young-Laplace pressure drop, $\Delta P_{\text {surf }}$, across it which is given by [19-21]

$\Delta P_{\text {surf }}=\frac{2 \gamma}{R}=\rho g h, \quad$ with $R=\frac{a}{\cos (\theta)}$,

where $R$ is the curvature radius of the meniscus, $\theta$ is the contact angle at liquid/solid/air interface, and $h$ is the equilibrium height of the liquid column ( $h<0$ in the case of capillary fall). Untreated PDMS
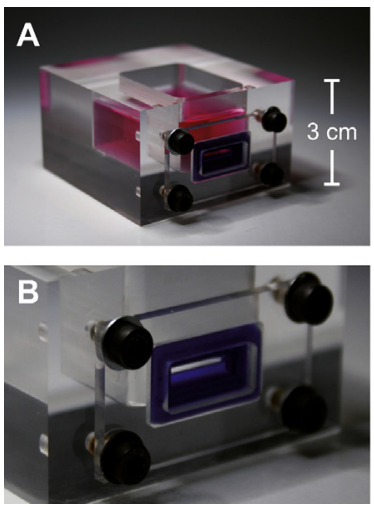
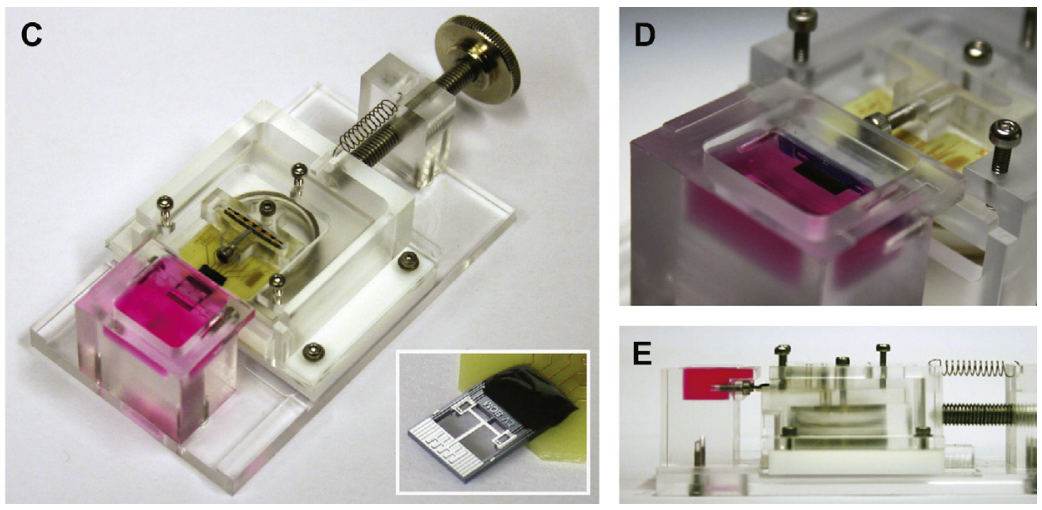

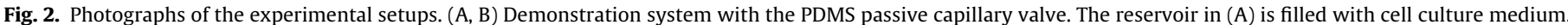

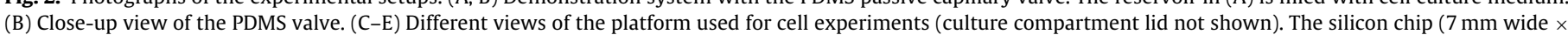
$9 \mathrm{~mm}$ long) is partially immersed in medium. The inset in (C) shows a magnification of the silicon chip. Opening dimension of the valve: $11 \mathrm{~mm}$ wide $\times 1 \mathrm{~mm}$ high. 
A
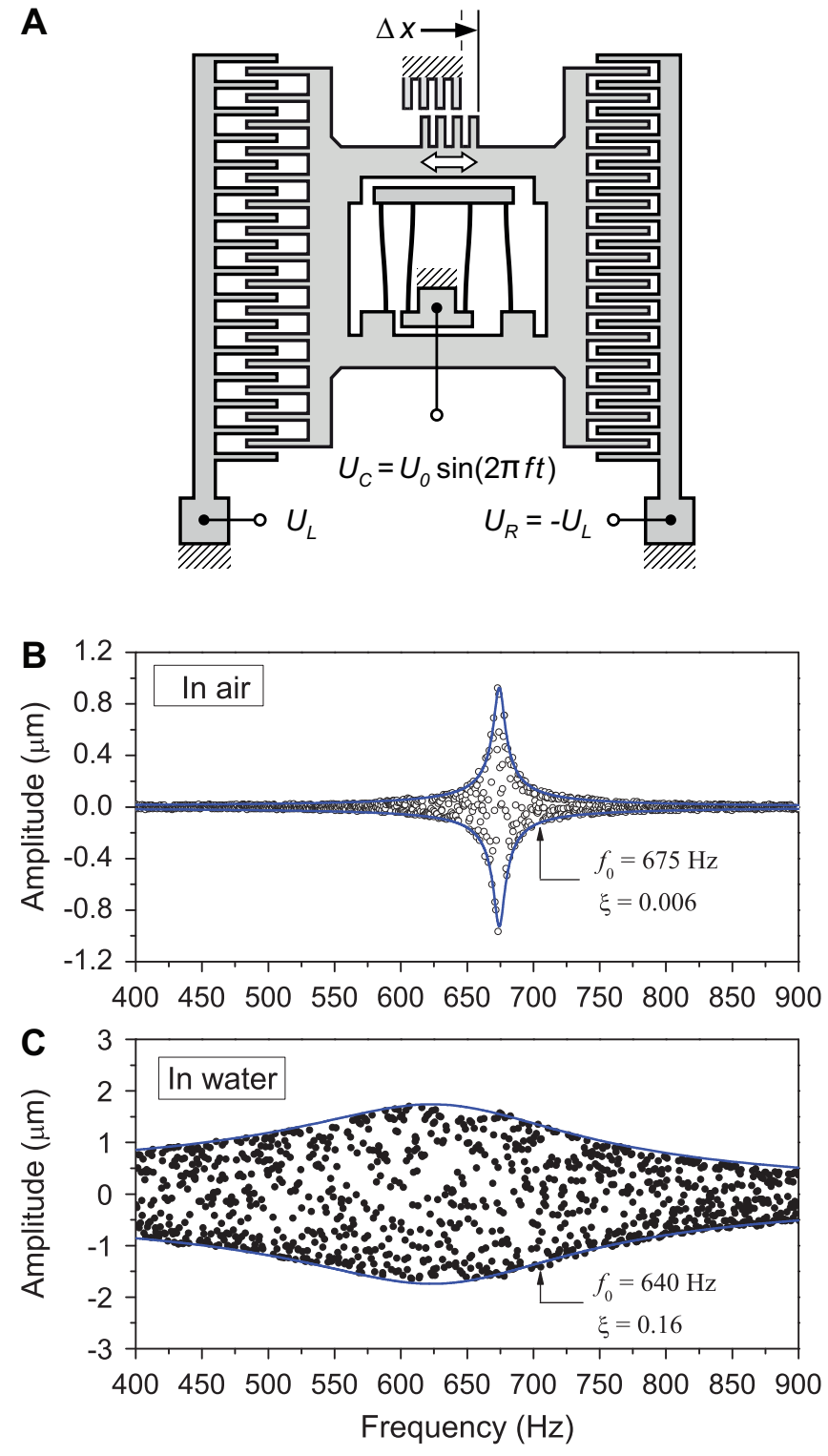

Fig. 3. Schematic of the push-pull electrostatic comb-drive actuator and dynamic characterization of a fabricated device. (A) Two dc power supplies are connected to the left and right fixed combs ( $U_{L}$ and $U_{R}$, respectively), while an ac power supply $\left(U_{C}\right)$ drives the central combs (sinusoidal signal of frequency $f$ ). The displacement $\Delta x$ is measured by "temporally aliased video microscopy" thanks to the periodic patterns $[25,26]$. (B, C) Typical frequency responses of a MEMS device (B) in air and (C) in water. It is a damped harmonic oscillator with medium dependent resonant frequency $f_{0}=\omega_{0} / 2 \pi$ and damping coefficient $\xi$.

is hydrophobic and has an advancing contact angle of $\theta \approx 110^{\circ}$ [23], leading to capillary fall $\left(\Delta P_{\text {surf }}<0\right)$.

The case depicted in Fig. 1C can be solved similarly. Consider a circular aperture of hydraulic diameter $D_{\mathrm{H}}=2 a$. From Eq. (2), the liquid height $(h>0$, see the sign convention in Fig. 1$)$ that the valve can withstand is

$h=-\frac{4 \gamma}{\rho g D_{\mathrm{H}}} \cos (\theta)$, with $\theta>90^{\circ}$.

From Eq. (3) we find that, for a $11 \mathrm{~mm}$ wide $\times 1 \mathrm{~mm}$ high rectangular opening in PDMS, the admissible liquid height is $h \approx 5.3 \mathrm{~mm}$. We have tested the setup shown in Fig. 2A and B with either DMEM or deionized water to measure the burst pressure. In all our experiments, the valves withstood larger hydrostatic pressures than expected: liquid burst occurred for $h \approx 10-15 \mathrm{~mm}$. This

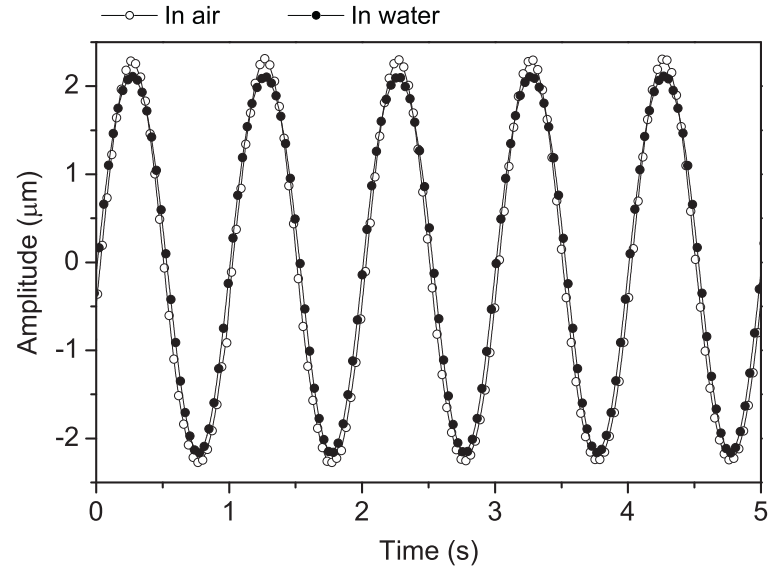

Fig. 4. Measurement of device oscillations in air and in water. For these experiments, the device was excited with a $1 \mathrm{~Hz}$ sinusoidal signal using identical actuation voltages. Water damping causes a decrease of the oscillation amplitude by about 5 $\%$.

difference may be explained by the roughness of valve sidewalls which increases the effective contact angle.

Young-Laplace pressure drop can also be estimated across plates with different contact angles [19]. This case occurs when a plate (thickness $e$, contact angle $\theta_{2}$ ) is introduced inside the PDMS valve (contact angle $\theta_{1}$ ), as illustrated in Fig. 1D. It causes the meniscus to reshape around the solid surfaces. In this paper, for the sake of simplicity, we have assumed that the pressure drop is only slightly modified when a silicon chip (with hydrophilic surfaces) is inserted in the capillary opening. Readers interested in such calculations may find useful information in works dealing with self-assembly or AFM-like probing $[14,15,23]$.

\subsection{MEMS device characterization}

The linear motion of the silicon device is obtained thanks to a push-pull electrostatic actuator [24]. With the driving scheme illustrated in Fig. $3 \mathrm{~A}$, the electrostatic force, $F_{x}(t)$, is proportional to the ac driving voltage, $U_{C}(t)$, and can be written [25]

$F_{x}(t)=F_{0} \sin (\omega t)$, with $F_{0}=2 \frac{\partial C}{\partial x} U_{0} \cdot U_{L}$,

where $t$ is the time, $\omega$ is the angular frequency of the ac signal, $U_{0}$ is the amplitude of the ac voltage, $U_{L}$ is the amplitude of the dc voltage, and $\partial C / \partial x$ is the spatial derivative of the comb-drive capacitance, which can be assumed constant for interdigitated comb electrodes and small displacements [24]. Hence, the amplitude of the driving force, $F_{0}$, is a constant and the equation of motion of the MEMS device is simply given by the linear differential equation [25]

$\ddot{x}+2 \xi \omega_{0} \dot{x}+\omega_{0}^{2} x=\frac{F_{0}}{M} \sin (\omega t)$,

where $x$ is the lateral displacement of the structure, $\xi$ is the damping coefficient which depends on the medium (air or liquid), $M$ is the effective mass of the moving structure, and $\omega_{0}$ is the undamped angular frequency.

In Fig. 3B and C, we show typical frequency responses of a MEMS device actuated in air and in water. These measurements were performed by "temporally aliased video microscopy" ${ }^{1}$, through

\footnotetext{
1 "Temporally aliased video microscopy" is an optical method used for the in plane dynamic characterization of MEMS with a conventional CCD camera. Using a digital microscope recording videos at a limited frame rate (typically $28 \mathrm{fps}$ ), despite undersampling, this method is efficient at extracting the frequency response functions of MEMS devices having resonant frequencies up to a few $\mathrm{kHz}$.
} 

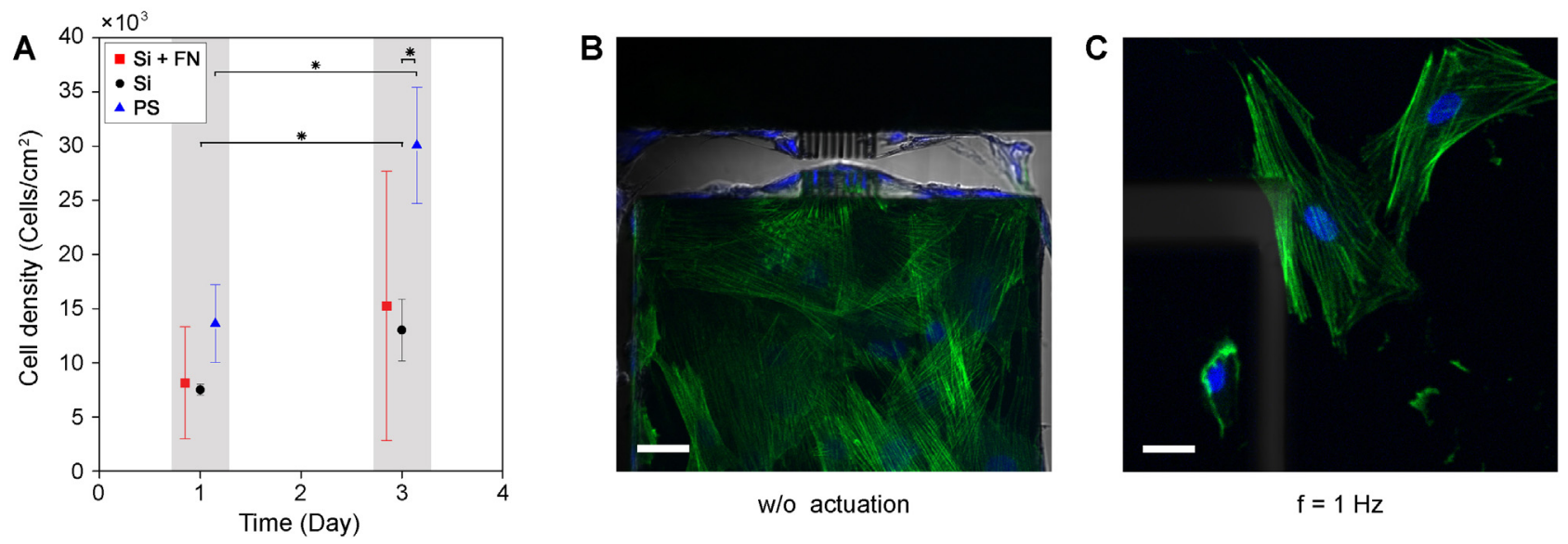

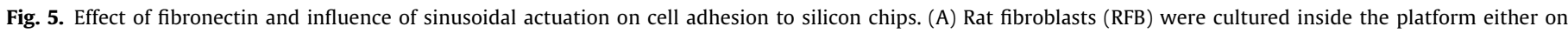

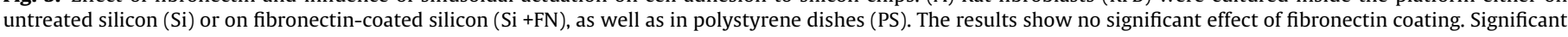
$P$-value: ${ }^{*} P<0.05$. (B, C) RFB were cultured inside the microengineered platform on untreated silicon before fixation for immunofluorescent imaging. Scale bar: $50 \mu m$.

analysis of the dynamic motion of periodic patterns etched in the silicon device $[25,26]$. For comparison purposes, these experiments were performed consecutively on the same packaged chip (see inset in Fig. 2C). They were observed under an upright digital microscope (VHX-600, Keyence Corp., Japan) using the setup shown in Fig. 2C-E, first without liquid, then after filling the fluidic chamber with deionized water. Because of damping, the resonant frequency decreased when the chip was partially immersed in water. However, for low frequencies (typically $\ll 100 \mathrm{~Hz}$ for mechanobiology assays), the influence of damping remained negligible. This can be verified in Fig. 4 where we show typical measurements performed on a chip excited at $1 \mathrm{~Hz}$ in air and in water under identical actuation voltages.

\subsection{Biological experiments}

Ensuring biocompatibility of bio-MEMS is a key issue for biological systems such as cells. Thus, we assessed RFB survival and proliferation inside the platform after 1 and 3 days. Within the same experiment, we also compared the adhesion of cells between untreated and fibronectin-coated silicon chips. However, the chips were not actuated for these experiments. In Fig. 5A, the graph shows that cells survived and grew in the platform for 3 days. The number of cells differs significantly between Petri dishes and silicon chips. This can be explained by the difference in surface properties [27]. On the other hand, no significant effect on cell adhesion was noticed between uncoated and fibronectin-coated silicon chips. We also observed a higher cell number variability on fibronectin-coated chips. This discrepancy can be explained by the weak binding forces between fibronectin and silicon. Therefore, the following experiments were performed with uncoated silicon chips.

Our ultimate goal being to use the platform for stretching and contraction assays on cells adhered to the substrate, RFB were cultured for 2 days inside the platform prior to the experiments. Signals between dc and $1 \mathrm{~Hz}$ were used to actuate the devices, while the amplitude and actuation duration $( \pm 5 \mu \mathrm{m}, 1 \mathrm{~h})$ were kept identical for all the experiments. Fig. 5B and $C$ show typical results observed on unactuated chips and chips actuated at $1 \mathrm{~Hz}$, respectively. A drastic cell detachment was observed as the actuation frequency was increased (results not shown). For $1 \mathrm{~Hz}$ excitation, only $10 \%$ of the cells remained attached to the chips. According to Couette flow theory [19], the shear stress sensed by the cells at $1 \mathrm{~Hz}$ can be estimated to be $\approx 2 \times 10^{-6} \mathrm{~Pa}$. Compared to the average shear strength required for cell detachment from glass (530-750 Pa) [28] or from silicon (80 Pa) [29], the calculated stress is 8 orders of magnitude lower. Thus, shear flow alone cannot explain cell detachment. It is more likely due to the weak binding forces between cells and the silicon substrate. Therefore, we believe that covalent patterning of fibronectin to silicon (e.g., through a silanization process [30]) would improve cell attachment.

We observed that cells tended to adhere preferentially on unstructured large surfaces, as well as on the periodic structures aimed for displacement measurements. As shown in Fig. 5B, these structures are separated by $<5 \mu \mathrm{m}$ gaps. On the other hand, the cells did not make the expected bridging over the $20 \mu \mathrm{m}$ wide gaps designed for that purpose. Referring to the recent work of Kuribayashi-Shigetomi et al. on bovine aortic smooth muscle cells, the gap for cell bridging should actually not exceed few micrometers, typically $\leq 8 \mu \mathrm{m}[31]$.

\section{Conclusion and outlook}

We have presented a proof of concept for a platform consisting of a fluidic device and an electrostatically actuated MEMS device. We could demonstrate the functionality of passive capillary valves over extended periods, as well as fibroblast viability inside the platform. Using immunofluorescent imaging, we have studied cell adhesion efficiency on uncoated and fibronectin-coated chips. In the latter case, coating did not improve cell adhesion due to the weak link between fibronectin and silicon. On uncoated silicon, we have observed drastic cell detachment, even for low actuation frequencies. Furthermore, in order to use the platform for stretching and compression assays, the design of the chip should be slightly modified: The gap between the mobile and the immobile part should not exceed $8 \mu \mathrm{m}$ [31]. Improving cell adhesion to silicon substrate as well as cell bridging across the microplates will be crucial for future cell-on-chip experiments. Once these two aspects will be optimized, stretching and contraction assays could be feasible.

We think that this platform will be a versatile instrument for cell mechanobiology. It can serve as a dedicated tool for biophysical experiments on adherent cells, with direct application to mechanotransduction assays on more complex and relevant cell types, such as stem cells.

\section{Acknowledgments}

This research was supported by the Swiss National Science Foundation through grants PZ00P2_139423 and CR32I3_130320.

We are thankful to the students who have contributed to this work: Marc-Olivier Bammatter and Etienne Lançon for the design 
of the silicon chip, Nicolas Beuchat and Yannick Devaud for their work on cell culture on silicon chips.

\section{References}

[1] D.-H. Kim, K.W. Pak, J. Park, A. Levchenko, Y.Sun, Microengineered platforms for cell mechanobiology, Annual Review of Biomedical Engineering 11 (1) (2009) 203-233, http://dx.doi.org/10.1146/annurev-bioeng-061008-124915.

[2] G.H. Altman, R.L. Horan, I. Martin, J. Farhadi, P.R. Stark, V. Volloch, J.C. Richmond, G. Vunjak-Novakovic, D.L. Kaplan, Cell differentiation by mechanical stress, FASEB Journal 16 (2) (2002) 270-272, http://dx.doi.org/10.1096/fj.01-0656fje.

[3] D.A. Lee, M.M. Knight, J.J. Campbell, D.L. Bader, Stem cell mechanobiology, Journal of Cellular Biochemistry 112 (1) (2011) 1-9, http://dx.doi.org/10.1002/jcb.22758.

[4] A.I. Caplan, S.P. Bruder, Mesenchymal stem cells: building blocks for molecular medicine in the 21st century, Trends in Molecular Medicine 7 (6) (2001) 259-264, http://dx.doi.org/10.1016/S1471-4914(01)02016-0.

[5] T.D. Brown, Techniques for mechanical stimulation of cells in vitro: a review, Journal of Biomechanics 33 (1) (2000) 3-14, http://dx.doi.org/10.1016/S0021-9290(99)00177-3.

[6] R. McBeath, D.M. Pirone, C.M. Nelson, K. Bhadriraju, C.S. Chen, Cell shape, cytoskeletal tension, and RhoA regulate stem cell lineage commitment, Developmental Cell 6 (4) (2004) 483-495, http://dx.doi.org/10.1016/S1534-5807(04)00075-9.

[7] A. Engler, S. Sen, H.L. Sweeney, D.E. Discher, Matrix elasticity directs stem cell lineage specification, Cell 126 (4) (2006) 677-689, http://dx.doi.org/10.1016/j.cell.2006.06.04.

[8] J.-C. Wang, G. Yang, Z. Li, Controlling cell responses to cyclic mechanical stretching, Annals of Biomedical Engineering 33 (3) (2005) 337-342, http://dx.doi.org/10.1007/s10439-005-1736-8.

[9] C. Moraes, Y. Sun, C. Simmons, (Micro)managing the mechanical microenvironment, Integrative Biology 3 (10) (2011) 959-971, http://dx.doi.org/10.1039/C1IB00056J.

[10] S. Yang, T. Saif, Micromachined force sensors for the study of cell mechanics, Review of Scientific Instruments 76 (4) (2005), http://dx.doi.org/10.1063/1.1863792, art. no. 044301.

[11] D.B. Serrell, T.L. Oreskovic, A.J. Slifka, R.L. Mahajan, D.S. Finch, A uniaxial bioMEMS device for quantitative force-displacement measurements, Biomedical Microdevices 9 (2) (2007) 267-275, http://dx.doi.org/10.1007/s10544-006-9032-4.

[12] M.A. Erismis, H. Pereira Neves, P. De Moor, R. Puers, C. Van Hoof, A water-tight packaging of MEMS electrostatic actuators for biomedical applications, Microsystem Technologies 16 (12) (2010) 2109-2113, http://dx.doi.org/10.1007/s00542-010-1136-3.

[13] E. Dy, C. Ho, Development of a cytomic force transducer for experimental mechanobiology, in: Proc. 22nd IEEE Int. Conf. Micro Electro Mech. Syst., Sorrento, Italy, 2009, pp. 391-394, http://dx.doi.org/10.1109/MEMSYS.2009.4805401.

[14] C. Yamahata, E. Sarajlic, L. Jalabert, M. Kumemura, D. Collard, H. Fujita, Mechanical characterization of biomolecules in liquid using silicon tweezers with subnanonewton resolution, in: Proc. 22nd IEEE Int Conf. Micro Electro Mech. Syst., Sorrento, Italy, 2009, pp. 607-610, http://dx.doi.org/10.1109/MEMSYS.2009.4805455.

[15] M. Kumemura, D. Collard, R. Tourvielle, N. Lafitte, K. Montagne, S. Yoshizawa, D. Fourmy, C. Yamahata, L. Jalabert, Y. Sakai, S. Takeuchi, T. Fujii, H. Fujita, Integrated MEMS platform with silicon nanotweezers and open microfluidic device for molecular and cellular biomechanical assays, in: Proc. 24th IEEE Int. Conf. Micro Electro Mech. Syst., Cancùn, Mexico, 2011, pp. 67-70, http://dx.doi.org/10.1109/MEMSYS.2011.5734363.

[16] B. Hinz, G. Celetta, J.J. Tomasek, G. Gabbiani, C. Chaponnier, Alpha-smooth muscle actin expression upregulates fibroblast contractile activity, Molecular Biology of the Cell 12 (9) (2001) 2730-2741

[17] M. Stranczl, E. Sarajlic, H. Fujita, M.A.M. Gijs, C. Yamahata, High-angular-range electrostatic rotary stepper micromotors fabricated with SOI technology, Journal of Microelectromechanical Systems 21 (3) (2012) 605-620, http://dx.doi.org/10.1109/JMEMS.2012.2189367.

[18] A. Desmoulière, L. Rubbia-Brandt, A. Abdiu, T. Walz, A. MacieiraCoelho, G. Gabbiani, Alpha-smooth muscle actin is expressed in a subpopulation of cultured and cloned fibroblasts and is modulated by gamma-interferon, Experimental Cell Research 201 (1) (1992) 64-73, http://dx.doi.org/10.1016/0014-4827(92)90348-C.

[19] H. Bruus, Theoretical Microfluidics, Oxford Master Series in Physics, Oxford University Press, Oxford, New York, 2008, Ch. 7.

[20] P.F. Man, C.H. Mastrangelo, M.A. Burns, D.T. Burke, Microfabricated capillarity-driven stop valve and sample injector, in: Proc. 11th IEEE Annu. Int. Workshop Micro Electro Mech. Syst., 1998, pp. 45-50, http://dx.doi.org/10.1109/MEMSYS.1998.659727.

[21] J.M. Chen, Analysis and experiment of capillary valves for microfluidics on a rotating disk, Microfluidics and Nanofluidics 4 (5) (2008) 427-437. http://dx.doi.org/10.1007/s10404-007-0196-x.

[23] M. Morra, E. Occhiello, R. Marola, F. Garbassi, P. Humphrey, D. Johnson, On the aging of oxygen plasma-treated polydimethylsiloxane surfaces, Journal of Colloid and Interface Science 137 (1) (1990) 11-24, http://dx.doi.org/10.1016/0021-9797(90)90038-P.
[23] T.A. Saif, On the capillary interaction between solid plates forming menisci on the surface of a liquid, Journal of Fluid Mechanics 473 (2002) 321-347, http://dx.doi.org/10.1017/S0022112002002483.

[24] W.C. Tang, T.-C.H. Nguyen, R.T. Howe, Laterally driven polysilicon resonant microstructures, Sensors and Actuators 20 (1-2) (1989) 25-32, http://dx.doi.org/10.1016/0250-6874(89)87098-2.

[25] C. Yamahata, M. Stranczl, E. Sarajlic, G.J.M. Krijnen, M.A.M. Gijs, Temporally aliased video microscopy: an undersampling method for in-plane modal analysis of microelectromechanical systems, Journal of Microelectromechanical Systems 21 (4) (2012) 934-944, http://dx.doi.org/10.1109/JMEMS.2012.2191265.

[26] C. Yamahata, E. Sarajlic, G.J.M. Krijnen, M.A.M. Gijs, Subnanometer translation of microelectromechanical systems measured by discrete Fourier analysis of CCD images, Journal of Microelectromechanical Systems 19 (5) (2010) 1273-1275, http://dx.doi.org/10.1109/JMEMS.2010.2067445.

[27] E.M. Harnett, J. Alderman, T. Wood, The surface energy of various biomaterials coated with adhesion molecules used in cell culture, Colloids and Surfaces B 55 (1) (2007) 90-97, http://dx.doi.org/10.1016/j.colsurfb.2006.11.021.

[28] A. Yamamoto, S. Mishima, N. Maruyama, M. Sumita, A new technique for direct measurement of the shear force necessary to detach a cell from a material, Biomaterials 19 (7-9) (1998) 871-879, http://dx.doi.org/10.1016/S0142-9612(97)00248-2.

[29] N.W. Karuri, S. Liliensiek, A.I. Teixeira, G. Abrams, S. Campbell, P.F. Nealey, C.J Murphy, Biological length scale topography enhances cell-substratum adhesion of human corneal epithelial cells, Journal of Cell Science 117 (15) (2004) 3153-3164, http://dx.doi.org/10.1016/j.biomaterials.2006.01.044.

[30] H. Majd, P.-J. Wipff, L. Buscemi, M. Bueno, D. Vonwil, T.M. Quinn, B. Hinz, A novel method of dynamic culture surface expansion improves mesenchymal stem cell proliferation and phenotype, Stem Cells 27 (1) (2009) 200-209, http://dx.doi.org/10.1634/stemcells.2008-0674.

[31] K. Kuribayashi-Shigetomi, H. Onoe, S. Takeuchi, Cell origami: self-folding of three-dimensional cell-laden microstructures driven by cell traction force, PLoS ONE 7 (12) (2012), http://dx.doi.org/10.1371/journal.pone.0051085, art. no. e51085.

\section{Biographies}

Tanja C. Hausherr was born in Vevey (Switzerland) in 1984. She completed her studies in Bioengineering at the Swiss Federal Institute of Technology (Ecole Polytechnique Fédérale de Lausanne, EPFL) with a Master thesis in the group of Prof. Matthias P. Lutolf (LSCB, EPFL) in 2011. After her M.Sc., she worked at the Laboratory of Microsystems (LMIS2, EPFL) as a Scientific Assistant from summer 2011 until spring 2012. She then joined the Laboratory of Biomedical Orthopedics (LBO, EPFL) as a PhD student.

Hicham Majd received his Master in Microengineering in 2002 and his PhD in Biotechnology and Bioengineering in 2008 from the Ecole Polytechnique Fédérale de Lausanne (EPFL, Lausanne, Switzerland). From 2002 to 2005, he worked as an R\&D engineer at EPFL and Cytomec Sàrl. There, he was responsible for the development of a prototype in a project involving a novel device for growing cells on highly extendable silicone surfaces. During his Ph.D. thesis, he has developed a novel polymer cell culture substrates for stem cells and tissue engineering applications. From 2008 to 2011, he worked as a project leader in University of Geneva, Centre Hospitalier Universitaire Vaudois (CHUV, Lausanne, Switzerland) and EPFL to develop innovative surface coating for biomedical implants. In parallel, he has co-founded Labseed Sàrl, a start-up company developing a strategy combining biology, engineering and microtechnology expertise to face a clinically relevant problem, the encapsulation of implanted medical devices.

Damien Joss obtained his degree in Microengineering at HE-Arc Engineering School of Le Locle, Switzerland, in 2007. He joined the Institute of Applied Microtechnologies (IMA-Arc, La Chaux-de-Fonds, Switzerland) as a Research Assistant to work on various projects in the microsystems, surface technologies and medical devices areas. In parallel, he completed his Master of Science in Biomedical Engineering a the Medicine Faculty of Bern University, Switzerland. In 2010, he was part of the Laboratory of Microsystems (LMIS) and of the Laboratory of Biomechanical Orthopedics (LBO) at the Swiss Federal Institute of Technology (EPFL, Lausanne, Switzerland) on an interdisciplinary research project. Since 2011, he pursues his career in the medica devices industry (ophthalmology and drug delivery systems) as Process and Materials Specialist and is currently working as a Project Leader on the development and manufacturing of combination products.

Arnaud Müller obtained his degree in Microengineering at the Swiss Federal Institute of Technology (Ecole Polytechnique Fédérale de Lausanne, EPFL) in 2011, where he achieved his Master Thesis in the Laboratory of Microsystems (LMIS). Since 2011 he works as development engineer and designs manufacturing and controlling equipment for silicon-wafer processing, with applications in the MEMS and photovoltaic industries.

Prof. Dominique Pioletti received his Master in Physics from the Swiss Federal Institute of Technology Lausanne (EPFL) in 1992. He pursued his education in the same Institution and obtained his Ph.D. in biomechanics in 1997. He developed original constitutive laws taking into account viscoelasticity in large deformations. Then he spent two years at UCSD as post-doc fellow acquiring know-how in cell and molecular biology. He was interested in particular to gene expression of bone cells in contact to orthopedic implant. From 2006 to 2013, he was an Assistant Professor at 
EPFL and since August 2013, was appointed Associate Professor of Biomechancis at EPFL. He is the director of the Laboratory of Biomechanical Orthopedics. His research topics include: Biomechanics of the musculoskeletal system; Mechano-transduction in bone; Development of orthopedic implant as drug delivery system; and Functional tissue engineering.

Prof. Martin A. M. Gijs received the B.Sc. and Ph.D. degrees in physics from the Katholieke Universiteit Leuven, Leuven, Belgium, in 1981 and 1986, respectively. In 1987, he joined Philips Research Laboratories, Eindhoven, The Netherlands. He has worked there on the microfabrication and nanofabrication processes of high-critical-temperature superconducting Josephson and tunnel junctions, the microfabrication of microstructures in magnetic multilayers showing the giant magnetoresistance effect, the design and realization of miniaturized motors for hard-disk applications, and the design and realization of planar transformers for miniaturized power applications. Since 1997, he has been with the École Polytechnique Fédérale de Lausanne, Lausanne, Switzerland, where he is currently a
Professor in the Institute of Microengineering and is responsible for the Microsystem Technology Group. His main interests are in developing technologies for novel magnetic devices, new microfabrication technologies for microsystem fabrication in general, and the development and use of microsystem technologies for microfluidic and biomedical applications in particular.

Christophe Yamahata obtained his degree in Microengineering from the Swiss Federal Institute of Technology Lausanne (Ecole Polytechnique Fédérale de Lausanne, EPFL) in 2000. He joined the Microsystems Laboratory (LMIS, EPFL) in 2001 as a Research Assistant where he completed a Ph.D. thesis on the topic of "Magnetically Actuated Micropumps" in 2005. From 2005 to 2008, he pursued research at the University of Tokyo, as a postdoctoral fellow of the Swiss National Science Foundation (SNSF) and later as a Japan Society for the Promotion of Science Postdoctoral Fellow postdoctoral fellow (2006-2008). From 2008 to 2012, he was a Research Associate at EPFL, funded by the SNSF under an "Ambizione" grant. He is a co-founder of VeoSwiss Technologies Sàrl. 